\title{
Not Returned
}

National Cancer Institute

\section{Source}

National Cancer Institute. Not Returned. NCI Thesaurus. Code C106464.

The status of an item that was not returned. 